\title{
DIGITALCOMMONS
}

@WAYNESTATE -

Wayne State University

Human Biology Open Access Pre-Prints

WSU Press

9-10-2013

\section{Intermittence for Humans spreading 45,000 years ago: from Eurasia to the Americas}

\author{
J.C. Flores \\ Universidad de Tarapacá, Arica, Chile, cflores@uta.cl
}

\section{Recommended Citation}

Flores, J. C., "Intermittence for Humans spreading 45,000 years ago: from Eurasia to the Americas" (2013). Human Biology Open Access Pre-Prints. Paper 38.

http://digitalcommons.wayne.edu/humbiol_preprints/38

This Open Access Preprint is brought to you for free and open access by the WSU Press at DigitalCommons@WayneState. It has been accepted for inclusion in Human Biology Open Access Pre-Prints by an authorized administrator of DigitalCommons@WayneState. 


\section{Intermittence for Humans spreading 45,000 years ago: from Eurasia to the Americas}

J. C. Flores (cflores@uta.cl)

Instituto de Alta Investigación IAI,

Universidad de Tarapacá, Casilla 7-D, Arica,

Chile.

Keywords: Population dynamics; Human dispersal; Archaeomath; Ancient migrations.

Running title: Intermittence and Human dispersal 


\begin{abstract}
From northeastern-Eurasia to the Americas, a three stage spread of Modern Humans is considered through large scale intermittence (exploitation/relocation). Conceptually, this work supports intermittence as a real strategy for colonization of new habitats. For the northeastern Eurasia travel, the first stage, we adapt our model to archaeological dates determining the diffusion coefficient (exploitation phase) as $D=299.44\left[\mathrm{~km}^{2} / \mathrm{yr}\right]$ and the velocity parameter (relocation phase) as $v_{o}=4.8944[\mathrm{~km} / \mathrm{yr}]$. The relative phaseweight $(\approx 0.46)$, between both kind of motions, is consistent with a moderate biological population rate $\left(r^{\prime} \approx 0.0046[1 / \mathrm{yrs}]\right)$. The second stage is related to population fragmentation. The last stage, reaching Alaska, corresponds essentially to relocation $\left(v_{o} \approx 0.75[\mathrm{~km} / \mathrm{yr}]\right)$.
\end{abstract}


In a general framework, scientists suggest the first Americans were Asian hunters following prey east-ward across Beringia, today, the submerged continent between Asia and Alaska. Starting from Asia, particularly Siberia around 45,000 years before present (BP), the expansion of Modern Humans to the Americas has been the subject of extensive debates, including genetic aspects (Reich et al 2012). In fact, a three-stage Modern Humans expansion process into the Americas (Alaska) has been conjectured (Kitchen et al 2008). That scheme integrates interdisciplinary studies including mathematical analysis among others. Particularly, the Fisher-Kolmogorov reactiondiffusion equation (Murray 2002, Benguria \& Depassier 2005) was used to support the three stage expansion from northeastern Eurasia to the Americas (Hamilton and Buchanan 2010). The respective wave-front solution represents a continuous advance without consider niche exploitation and relocation as two independent phases. For reviews and criticisms of reaction diffusion equations applied to archaeological problems see the article by Steele (2009). For applications to Language competition, population growth and dispersal see Kandler 2009. Others applications of wavefront spreads, for instance in the Paleolithic or Neolithic, could be found in Fort et al (1999, 2004) and Flores (2011) related to Neanderthals extinction.

As a new mathematical tool, aside of reaction diffusion equations, recent researches (Bénichou et al, 2006, 2011) have showed that the optimal process of resources searching (for instance, food) requires two phases, or levels,: (a) a purely diffusive motion (niche exploitation) and (b) a constant speed motion (relocation). It corresponds to a strategic mechanism for searching new targets. In this work, we shall consider these two integrated levels of spread, at large scale, rather than a wavefront point of view to describe ancient Human migrations. Critical reviews about the dispersion in to the Americas could be found in Rothhammer and Dillehay (2009), Maschner (1997) and Reich (2012). Finally, note that the more ancient dates for the Americas occupation correspond to Monte Verde (Chile) around 14,600 years BP (Fiedel 2002) and Buttermilk Creek with (possible) 15,500 years BP (Walters et al, 2011).

In section 1, the main tool to describe the elapsed time related to dispersal is introduced (diffusion/relocation). In section 2, the spread from Eurasia to the Americas is studied and the parameters (diffusion coefficient, velocity, phase-weight, etc.) are evaluated according to archaeological dates. In section 3, a connection between the usual growth 
rate (without relocation) and this one related to intermittence is conjectured and, applied to the ancient travel through northeastern Eurasia.

\section{1. - Exploitation-relocation as a large scale searching process}

Figure 1 shows schematically the above mentioned intermittence spread process related to niche exploitation and relocation. Further, this geographical intermittence becomes correlated to a biological one. The phase, or level, of the niche exploitation becomes accompanied with a population growth and, opposite, relocation correlates to a poor growth. In this sense, the integrated exploitation/relocation motion has an effective growth rate $r^{\prime}$ smaller than the purely exploitation-level with rate $r$ (section 3).

There are three basic parameters describing this process with intermittence: (i) The diffusion coefficient $D$, (ii) the positive velocity parameter $v_{o}$ and (iii) the characteristic time $\tau_{s}$ related to natural events like periodic annual season, generational time, or others. These three basic parameters are mathematically correlated (Bénichou et al, 2006) and, with some approximations, they have an intrinsic dependence:

$$
\tau_{s}=2 \frac{D}{v_{o}^{2}} .
$$

Note that, Fisher-Kolmogorov wave front solution also has similar parameters, but is a different kind of dispersal (constant speed). A straightforward way to consider exploitation/relocation is mathematical superposition (Flores, 2007). Thus, the elapsed time $\tau$ of spread becomes a quadratic function of the distance $x$. That is, $\tau=\alpha\left(x^{2} / D\right)+(1-\alpha)\left(x / v_{o}\right)$ or according to equation (1):

$$
\frac{\tau}{\tau_{s}}=2 \alpha\left(\frac{x}{\tau_{s} v_{o}}\right)^{2}+(1-\alpha)\left(\frac{x}{\tau_{s} v_{o}}\right) .
$$

Equation (2), the main tool used in this work, describes the elapsed time due to resources exploitation and relocation. The dimensionless parameter $\alpha$ determines the relative phase-weight, or influence, between both kind of motion and ranges as $0 \leq \alpha \leq 1$. When $\alpha \approx 1$, there is a predominant phase of niche-exploitation and consequently a rapid population enlargement. Opposite, when $\alpha \approx 0$, relocation predominates and low population growth is expected. 
Instantaneous velocity $v(\tau)$ has not a constant value when $\alpha \neq 0$, as occur for wavefront solutions, and usually $v \neq v_{o}$. This fundamental point makes a marked difference respect to usual spread modeling through reaction-diffusion equations. In fact, the instantaneous velocity defined as $v=d x / d \tau$, according to equation (2), becomes

$$
v=v_{o} \frac{1}{\left(4 \alpha x / v_{o} \tau_{s}\right)+(1-\alpha)} .
$$

Thus, the instantaneous velocity diminishes with the distance due to the temporal slow exploitation phase. Mathematically, it decreases since in the linear superposition (2), for long distance, the diffusive phase becomes dominant. From a migratory point of view, it is related to geographic connectivity and the probability to comeback, for instance, to the departure point. It includes the inverse route through relocation-pathway. In sections 2 and 3, we shall apply these concepts to the migration of Modern Humans from Eurasia to the Americas (Alaska).

Finally, note that two-phase ancient migration strategy have been yet considered qualitatively for Clovis, also conjectured for island-hopping on the early Polynesian Lapita people and the linearbandkeramik people (Fiedel 2002, for a review).

\section{2. - From Eurasia to the Americas: Results from archaeological dates}

Figure 2 shows the calibrate dates settlements (squared points) from Siberia to Alaska and discussed widely by Hamilton \& Buchanan (2010). They correspond to: Kara-Bom and Kara-Tenesh; Kandabaevo and Podzvonkaya; Zalainuoer; Ust-Mil2; Yana RHS; Ogonki 5; Siberdik; Ushkl; and finally Swan Point. Respect to the extreme points, the old one corresponds to Kara-Bom (Siberia) and the more recent to Swan Point (Alaska).

As conjectured by Kitchen et al (2008), there are three kinds of stages, or steps, in the mentioned migratory travel. They are marked, approximately, in the Figure 2 (region I, II, and III). To consider exploitation-relocation as a real strategy, the characteristic time $\tau_{s}$ is assumed as a generational one. Explicitly, $\tau_{s}=25$ [years] (Fort et al, 1999). 
In region I, the curve was adjusted directly according to equation (2). It corresponds to the best quadratic function adjusted through Mathematica6 tools $(\Delta \tau=0.109349 x+$ $0.00155222 x^{2}$ ). Numerically, one obtains the velocity parameter (not real velocity) $v_{o}=4.8944[\mathrm{~km} / \mathrm{yr}]$ and the corresponding diffusion coefficient becomes $D=299.44\left[\mathrm{~km}^{2} / \mathrm{yr}\right]$ in this stage. Moreover, through the same numerical analysis, the relative weight parameter becomes $\alpha=0.46480$ supporting intermittence across the northeastern region of Eurasia (region I). Plausibly, with this value for the phase-weight parameter, a moderate population growth turns out to be projected (section 3 ). In general, and as conclusion, this region (I) is well described by the quadratic function defined by equation (2). It conceptually supports intermittence strategy as a positive model for Human spread at large scale.

Region II becomes essentially related to fragmentation (isolation) and not migration occurs. There are data fragmentation (Beringia settlement) and apparently genetic diversification (Kitchen et al, 2008). This population partition was pushed by the Last Glacial Maximum around 20,000 years BP.

Region III corresponds to a spread process essentially related to relocation, as one sees from the data (Figure 2), possibly due to the post Last Glacial Maximum changes. Numerically the velocity parameter is estimated as $v_{o} \approx 0.75[\mathrm{~km} / \mathrm{yr}]$ and $\alpha \approx 0$. Eventually, this continuous motion could be associated to a wavefront of constant speed. It is similar, for instance, to this one of Paleolithic hunter-gathering in the northwest Europe, after the Last Glacial Maximum, with 0.5-2 [km/yr] (see Chamberlain 2009, and references therein).

\section{3. - Modified growth rate connected to the phase-weight}

In population dynamics, for instance Verhulst's equation (Murray 2002), the growth rate $r([1 / \mathrm{yr}])$ measures population variations and, in our migratory schema, becomes related essentially to exploitation of a given niche. Quantitatively, it corresponds to the rate of births minus deaths. Nevertheless, the integrated motion described by equation (2) incorporates intrinsically relocation. Thus, the effective growth rate $r^{\prime}$ (related to exploitation/relocation) must be different from the usual one $(r)$. Since in the purely 
relocation phase ( $\alpha \approx 0$ ) a low population growth is expected, the modified rate $r^{\prime}$ must be proportional to the usual one. That is,

$$
r^{\prime}=\alpha r, \quad \text { (effective rate) }
$$

We emphasizes that equation (4) describe an integrated process (exploitation/relocation) rather than a purely scheme of exploitation with rate $r$.

In this article, we shall consider a typical value for ancient populations as $r \approx 0.01$ (Fort et al 1999, Flores 2011). Therefore, for the intermittent spread in the northeastern Eurasia (region I), we have an effective rate $r^{\prime}=0.46148 \times 0.01=0.0046148[1 / \mathrm{yr}] \mathrm{a}$ quite moderate growth rate for this migration process (region I).

Finally, two considerations: (a) A dynamical equation describing the population growth, for instance Verlhust, contains the rate $r$ as a parameter (Murray 2002). Thus, through equation (4), the intermittence process (2) and the corresponding population equationgrowth become coupled. (b) In the purely relocation phase ( $\alpha \approx 0)$, the motion is essentially related to a constant speed, plausibly, also this relocation-phase could be modeled through a wave-front scheme.

\section{Conclusions and discussions}

The three step spread of Moderns from northeastern Eurasia to Americas was considered in the frame work of (large scale) intermittence (2). The first stage $(48,000-$ $30,000$ years BP, approx.) was described through intermittence ( $\alpha=0.46148)$. It is consistent with a moderate population growth $\left(r^{\prime}=0.0046148[1 / \mathrm{yr}]\right)$ and a conceptual confirmation that exploitation-relocation, rather than a continuous wavefront motion, occurred. The second stage (30,000-18,000 BP, approx.) is quite partitioned and consistent with genetic human diversification. The last stage (18,000-15,000 BP, approx.) essentially corresponds to a migratory motion in the relocation phase $\left(v_{o} \approx 0.75[\mathrm{~km} / \mathrm{yr}], \alpha \approx 0\right)$.

Finally, we have used the average generational time of 25 years. Eventually, other values could be considered, for instance $20<\tau_{s}<30$. Figures (3a) and (3b) show the 
curves for other values of $\tau_{s}$. The diffusion coefficient $D$ and the phase-weight $\alpha$ seem not very sensitive to changes around 25 years. In fact, the maximum value for the diffusion becomes $D_{\max } \approx 644\left[\mathrm{~km}^{2} / \mathrm{yr}\right]$.

Acknowledgements: This work was supported by project Fondecyt \# 1120344.

\section{References}

Benguria, R. D. \&. Depassier, M. C, 2005, Variational Principles for the Speed of Traveling Fronts of Reaction-Diffusion Equations, in Variational and Extremum Principles in Macroscopic Systems (S. Sieniutycz, ed., Elsevier Science, 339-352).

Bénichou, O. Coppey, M. Moreau, M. \& Voituriez, R. 2006, Intermittent research strategies: when losing time becomes efficient. Europhys. Lett. 75, 349.

Bénichou, O. Loverdo, C. Moreau, M. and Voituriez, R. 2011. Intermittent search strategies. Reviews of Modern Physics 83, 81.

Chamberlain, A. 2009, Archaeological demography. Human Biology 81, 275-286.

Fiedel, S. J., 2002, Initial Human colonization of the Americas: an overview of the issues and the evidences. Radiocarbon 44, 407-436. 
Flores, J. C. 2007, Dispersal time for ancient human migrations: Americas and Europe colonization. Europhys. Lett. 79, 18004.

Flores, J. C. 2011, Diffusion coefficient of Modern Humans outcompeting Neanderthals. Journal of Theoretical Biology 280, 189-190. Also, 1998, Journal of Theoretical Biology 191, 295-298.

Fort, J. and Méndez V. 1999, Time-delay theory of the Neolithic transition in Europe. Phys. Rev. Lett. 82, 867-870.

Fort, J. Pujol, T. and Cavalli-Sforza, L.L. 2004. Palaeolithic populations and wave of advance. Cambridge Archaeological Journal, 14, 53-56.

Hamilton, M. J. Buchanan, B. 2010. Archaeological support to the three-stage expansion of the modern human across northeastern Eurasia and into the Americas. PLoS ONE 5(8): e12472.

Kandler, A. 2009. Demography and language competition. Human Biology 81, 181210.

Kitchen, A. Miyamoto, M. M. Mulligan, C. J. 2008. A three stage colonization model for the peopling of the Americas, PLoS ONE 3(2): e1596.

Maschner, H. D. G. 1997. American beginnings' and the archaeological record of Beringia: A comment on variability. Antiquity 71, 223-228. And references therein.

Murray, J. D. 2002. Mathematical Biology (Vol. I and II, Springer-Verlag, Third edition).

Reich D. et al. 2012, Reconstructing Native American Population History. Nature 488, 370-374.

Rothhammer F. and Dillehay T.D., 2009. The late Pleistocene colonization of South America: An interdisciplinary perspective. Annals of Human Genetics, 73, 540-549. 
Steele, J. 2009, Human Dispersals: Mathematical Models and the archaeological record. Human Biology, 81, 121-140.

Waters M. R., et al. 2011, The Buttermilk Creek Complex and the origin of Clovis at the Debra L. Friedkin site, Texas. Science 331, 1599-1603. 


\section{Figure captions}

Figure 1

A representative draw of intermittence geographical dispersal composed of niche exploitation (diffusion $D$ ) and relocation (velocity $V o$ ), both parameters are connected through Eq. (1). Equation (2) represents this large scale motion with relative weight, or influence, $\alpha$ for the diffusive motion and $(1-\alpha)$ for relocation.

Figure 2

Squared points represent dates for settlements from northeastern Eurasia to Alaska (Hamilton \& Buchanan 2010). The three regions correspond to different kind of spread. The continuous curve matches the intermittence model (equation (2)). The first region agrees basically to $\alpha=0.46148$ and a moderate population growth. The second region is connected to fragmentation and the third becomes linked to relocation $\left(v_{o} \approx 0.75[\mathrm{~km} / \mathrm{yr}], \alpha \approx 0\right)$. See section 3 for details.

Figure $3 a$

Diffusion coefficient $D\left[\mathrm{~km}^{2} / \mathrm{yr}\right]$ as function of $\tau_{s}$ (stage I). The vertical dashed line corresponds to the time-value used in this work (25 years) furnishing $D=299.44$ $\left[\mathrm{km}^{2} / \mathrm{yr}\right]$, nevertheless, others values should be envisaged depending on the choice of $\tau_{s}$. The maximum diffusion coefficient becomes $D_{\max } \approx 644\left[\mathrm{~km}^{2} / \mathrm{yr}\right]$.

Figure $3 b$

Relative phase-weight $\alpha$ as function of $\tau_{s}$. The vertical line corresponds to 25 years ( $\alpha=0.46148$ ). In general, this parameter ranges from zero to one. The negative values, or these larger than one, are fictitious solutions obtained from the figure 2. 

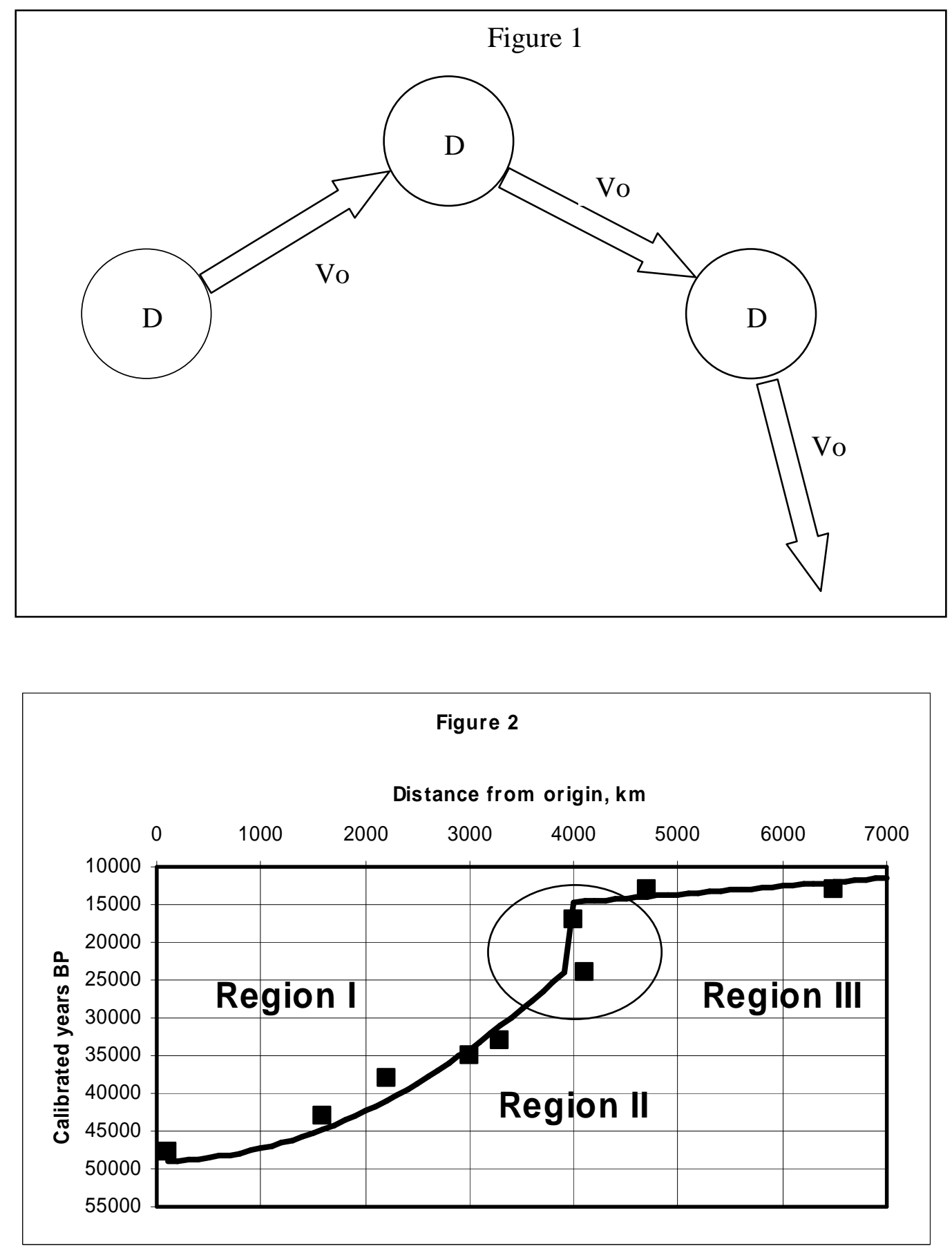
Figure3a

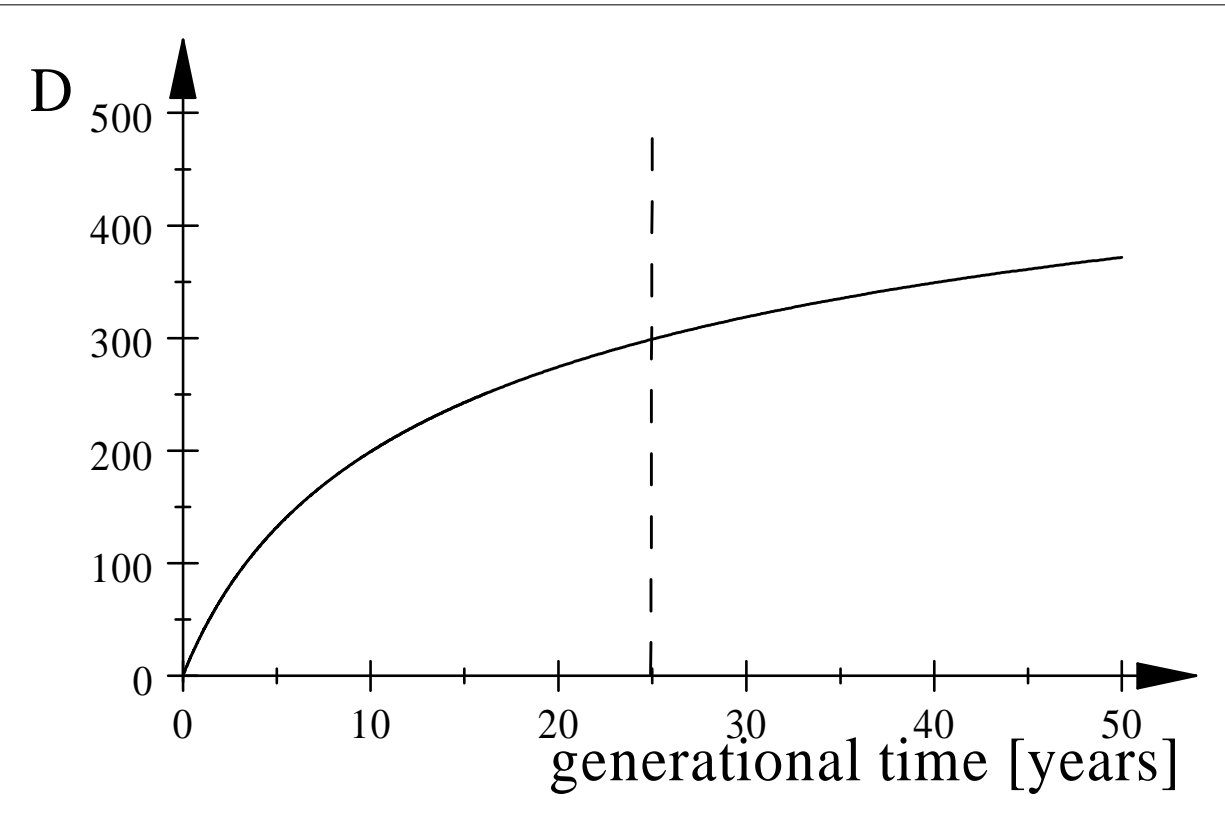

Figure $3 b$

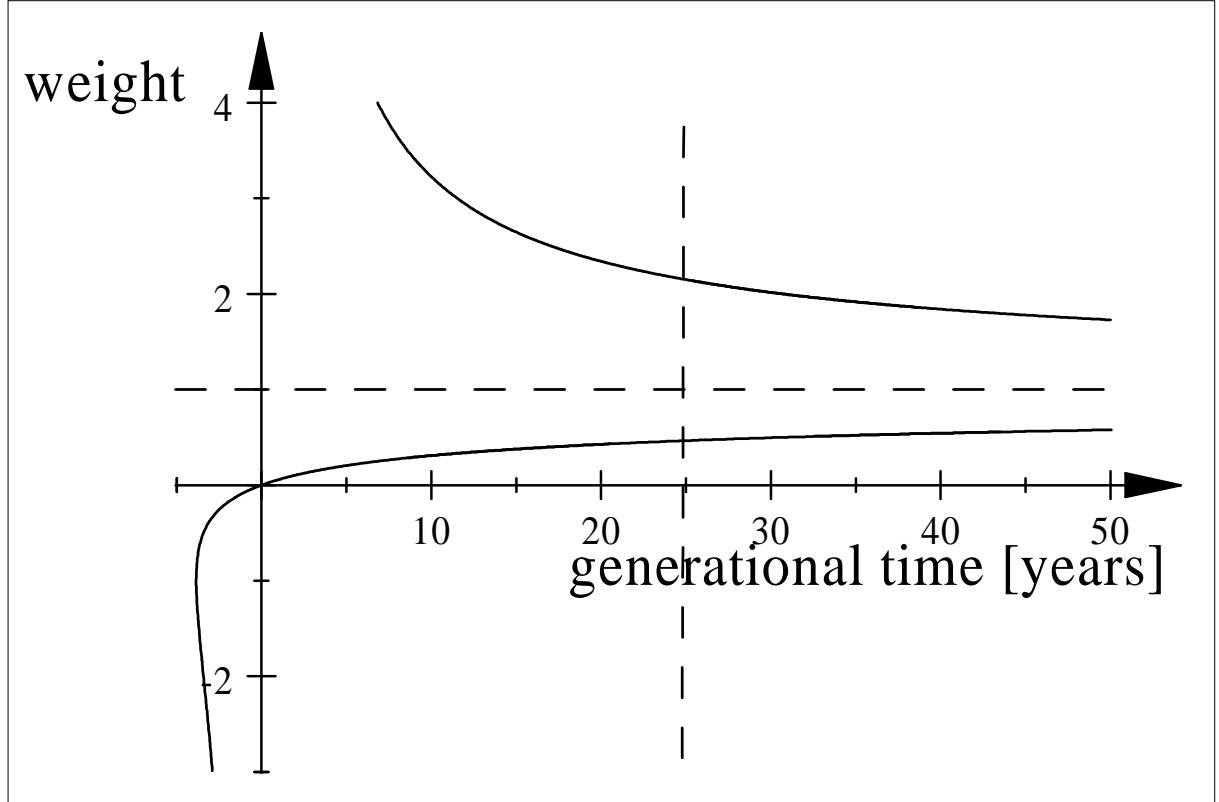

